gate is planar in the ordinary case, Fig. 1(f), with a uniform oxide thickness $a$, whereas in the proposed structure, Fig. 1(g), the gate has a circular front of radius r. Assuming a built-in charge density $Q$ at the surface and neglecting the space charge in the bulk, expressions of $I, V_{\theta}$, and $g_{m}$ can be found in the low $V_{d}$ region. The results are shown in the table, where $\epsilon_{0}$ is the permittivity of the insulator, $b=\sqrt{a^{2}-r^{2}}$ and again $\lambda=1-\delta$ with $\delta=0$ as the cutoff condition. For the case $(g), g_{n}$ has the following complicated form

$$
g_{m}=\frac{\epsilon_{0} \mu z V_{d}}{2 b^{2} \sqrt{\delta} \cosh ^{-1} \frac{a}{r}\left(\frac{L \sqrt{\delta}}{b}+\lambda \tan ^{-1} \frac{L}{b \sqrt{\delta}}\right)^{2}}\left[\frac{L b \lambda \sqrt{\delta}}{L^{2}+b^{2} \delta^{2}}+(2-\lambda) \tan ^{-1} \frac{\mathrm{L}}{b \sqrt{\delta}}\right]
$$

which, as $\delta \rightarrow 0$, is simplified to

$$
g_{m} \rightarrow \frac{\epsilon_{0} \mu z V_{d}}{\pi b^{2} \sqrt{\delta} \cosh ^{-1} \frac{a}{r}} .
$$

Equation (4) is seen to be similar to (2) in that $g_{m}$ varies reciprocally with $\sqrt{\delta}$.

The features of the proposed structures are demonstrated in Fig. 2 where $I$ is plotted vs. $V_{o}$ near the cutoff condition for both the unipolar and the surface FETs using $L=2 a$ and $r=a / 2$. The scales are normalized and the cut-off gate voltage $V_{g c}$ is chosen as the origin of the abscissa. At a given $V_{0}$ from its cutoff value, the current is higher in the cases (e) and (g) than (a) and (f); and at the origin, the slopes are finite in the cases (a) and ( $f$ ) but approach infinity in (e) and $(\mathrm{g})$. The infinite $\mathrm{g}_{m}$ arises mainly from our consideration of an infinitesimal signal and at $T=0^{\circ} \mathrm{K}$ so that the Debye transition

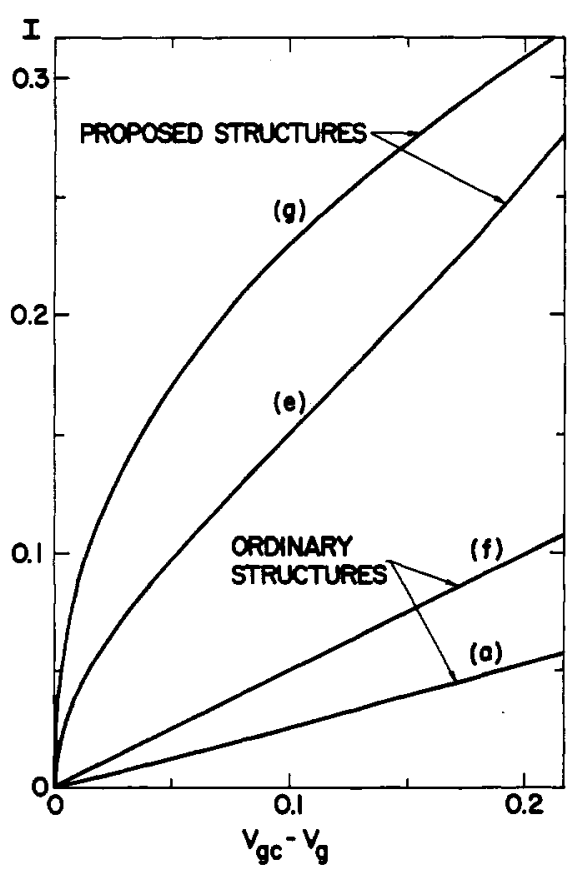

Fig. 2. Calculated characteristics of source-drain current vs. the deviation of applied gate voltage from cutoff gate voltage. The current and voltage scales are normalized, for unipolar FETs, cases (a) and (e), in pus $V_{d}$ and $\rho a^{2} / 2 \epsilon_{k}$; and for surface FETs, cases (f) and (g), in $Q_{\mu \nu} V_{d} / 2 a$ and $2 a / \epsilon_{0}$. width at the space charge boundary is zero. It is seen in the Figure, however, that the proposed structures will give notably higher $g_{m}$ than the ordinary structures at comparable gate capacitance. ${ }^{9}$

The $I-V_{d}$ relation cannot be readily derived, but is expected to follow the general behavior. That is, as $V_{d}$ increases $I$ increases with a decreasing slope, and finally becomes saturated as $V_{d}$ reaches its "pinch-off" value. The pinch-off voltage, probably fairly close to $V_{g c}-V_{g}$ shown in Fig. 2, is smaller in the cases (e) and (g) than (a) and (f) at the same

(saturated) current. This feature of low: voltage saturation is favorable in most device operations.

Our analyses were based on simplified assumptions in order to bring out the main features of the proposed structures. The gate actually may be placed anywhere between source and drain and may have other geometrical shapes (elliptical, for example). Furthermore, this type of gate may be combined in use with auxiliary planar gates to achieve various characteristics. In any case, the proposed devices should be operated near the cutoff condition so that a small signal at the gate would give a large variation in $I$ and a small voltage at the drain would saturate the current. These features together with the obvious feature of the minimal channel resistance to charge the gate capacity would promise their use in high-speed operations.

L. ESAKI

L. L. Chang

IBM Watson Research Center Yorktown Heights, N. Y.

9 The capacitance can be shown to be finite and comparable to that of the ordinary planar structure.

\section{On the Statistics of the Product of a Gaussian Noise Process and a Pseudorandom Binary Code}

In dealing with pseudorandom code ranging systems, used to track deep-space probes, one encounters a mechanism wherein a band-limited Gaussian channel noise process is multiplied by a pseudorandom binary ranging code. Since the statistics of the code and the noise are independent, the autocorrelation function of the product is the product of the autocorrelation functions of the two processes, and the noise spectral density of the product is easily derivable using the Fourier transform. However, a

Manuscript received October 4, 1965. question remains as to whether the statistics of the product remain Gaussian. The following demonstration is intended to show that the statistics of the product are indeed Gaussian.

The product is defined to be a stationary stochastic process, $Z(t)$ given by

$$
Z(t)=C(t) X(t)
$$

where $X(t)$ is a stationary random Gaussian noise process, and $C(t)$ is a pseudorandom binary waveform, taking on only values plus or minus one in a pseudorandom sequence, and having a fixed binary element length.

The function of interest is the probability distribution of the product, defined as

$$
F_{Z}(z)=P[Z \leq z] ; \quad Z=C X
$$

where $Z, C$ and $X$ are random variables and $z$ is a real number. The probability, $P$ is viewed as the probability of the "event" that the random variable $Z$ is less than or equal to the real number $z$. The event is a Boolean combination of events given as

$$
\begin{array}{r}
{[Z \leq z]=[Z \leq z] \cdot[C=+1] V[Z \leq z]} \\
\cdot[C=-1]
\end{array}
$$

where "." is the Boolean "and," "V" is the Boolean "or." Then,

$$
\begin{aligned}
F_{Z}(z)=P\{[Z \leq z] \cdot[C= & +1] V[Z \leq z] \\
& \cdot[C=-1]\} .
\end{aligned}
$$

Since the events $C=+1$ and $C=-1$ are mutually exclusive,

$$
\begin{aligned}
F_{Z}(z)= & P\{[Z \leq z] \cdot[C=+1]\} \\
& +P\{[Z \leq z] \cdot[C=-1]\} .
\end{aligned}
$$

Substituting from (1),

$$
\begin{aligned}
F_{Z}(z)= & P\{[X \leq z] \cdot[C=+1]\} \\
& +P\{[-X \leq z] \cdot[C=-1]\}
\end{aligned}
$$

The variables $X$ and $C$ are statistically independent so that

$$
\begin{aligned}
F_{\boldsymbol{Z}}(z)= & P[X \leq z] P[C=+1] \\
& +P[-X \leq z] P[C=-1] .
\end{aligned}
$$

Now the random variable $X$ is Gaussian and the probability that $X \leq z$ is the Gaussian distribution function, defined as $\Phi_{X}(z)$.

$$
\begin{aligned}
P[X \leq z] & \stackrel{\Delta}{=} \Phi_{X}(z) \\
P[-X \leq z] & =P[X \geq-z] \\
& \equiv 1-\Phi_{X}(-z)
\end{aligned}
$$

Then

$$
\begin{aligned}
F_{Z}(z)= & \Phi_{X}(z) P[C=+1] \\
& +\left[1-\Phi_{X}(-z)\right] P[C=-1] .
\end{aligned}
$$

Now, the Gaussian distribution function may be related to the "error function" as

$$
\Phi_{X}(z)=\frac{1}{2}\left[1+\operatorname{erf}\left(\frac{z}{\sqrt{2}}\right)\right] .
$$

Then

$$
\begin{gathered}
F_{Z}(z)=\frac{1}{2}\left[1+\operatorname{erf}\left(\frac{z}{\sqrt{2}}\right)\right] P[C=+1] \\
+\left\{1-\frac{1}{2}\left[1+\operatorname{erf}\left(\frac{-z}{\sqrt{2}}\right)\right]\right\} \\
P[C=-1] .
\end{gathered}
$$

Since

$\operatorname{erf}(-U)=-\operatorname{erf}(U)$ 


$$
\begin{gathered}
F_{Z}(z)=\frac{1}{2} P[C=+1]\left[1+\operatorname{erf}\left(\frac{z}{\sqrt{2}}\right)\right] \\
+\frac{1}{2} P[C=-1]\left[1+\operatorname{erf}\left(\frac{z}{\sqrt{2}}\right)\right] .
\end{gathered}
$$

But

$$
P[C=+1]+P[C=-1] \equiv 1 .
$$

Then

$$
\begin{array}{r}
F_{Z}(z)=\frac{1}{2}\left[1+\operatorname{erf}\left(\frac{z}{\sqrt{2}}\right)\right] \\
F_{Z}(z) \equiv \Phi_{X}(z) .
\end{array}
$$

It is seen that the probability distribution function of the product process $Z(t)$ is identically that of the Gaussian noise process, $X(t)$.

J. H. Painter

Motorola Inc.

Military Electronics Division Scottsdale, Ariz.

\section{A Circuit Model of the Step- Recovery Diode}

The charge-storage or step-recovery diode described by Moll et al. [1] has become a device of great interest, particularly in the generation of microwave power by highorder harmonic multiplication [2], [3]. For purposes of analysis and experimental scaling, it is useful to have a simple circuit model available which can accurately predict the performance of devices using these diodes. It is the purpose of this correspondence to present such a representation, which utilizes conventional capacitance and resistance in a piecewise linear model.

One of the basic characteristics of the step-recovery diode is its ability to store significant charge under forward bias. This stored charge is recoverable, and, hence, under forward bias the diode behaves much like a relatively large capacitance. But there is also a steady-state current under forward bias, as in a conventional diode, which requires the presence of a resistive component. A possible circuit model for a steprecovery diode under forward bias or charge storage conditions is, therefore, a simple parallel combination of a resistor and a capacitor. The validity of this approach can be checked by comparing the results obtained from the proposed model with (23) and (24) of Moll et al. These two equations pertain to the charge-storage phase of a diode which has been in forward conduction for time $T_{f}$, with a constant forward current $I_{f}$. At time $t=0$, the current is reversed in direction and held at magnitude $I_{r}$. The analogous situation for the proposed piecewise linear model is the circuit shown in Fig. 1(a). Under the condition of $R_{1} \ll R_{0}$, the forward

Manuscript received October 8, 1965. This work was supported in part by Watking-Johnson Co. Palo (PMR).

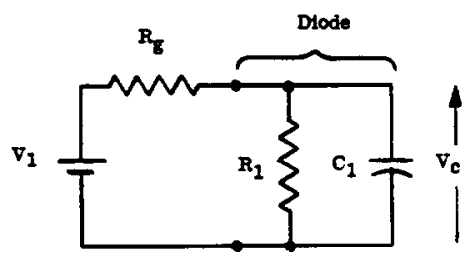

(a)

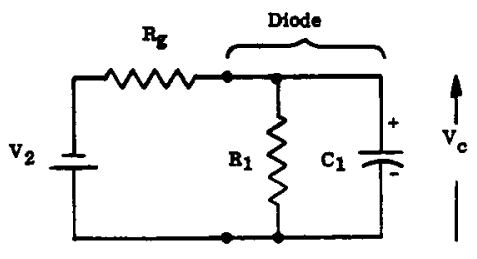

(b)

$$
\begin{aligned}
& \qquad \mathbf{R}_{\mathbf{I}} \ll R_{\mathbf{g}} \\
& \text { Fig. 1. A circuit representation of the step-recovery diode for } V_{c}>0 \text {. In (a), forward } \\
& \text { current } I_{i}=V_{1} / R_{0} \text { flows; in }(\mathrm{b}) \text {, reverse current } I_{r}=V_{2} / R_{q} \text { flows. }
\end{aligned}
$$

current will be constant at the value $I_{f}$ $=V_{1} / R_{g}$. Assuming at time $t=0$ there is no charge on the capacitor, the voltage of the capacitor as a function of time will be of the form

$$
V_{c}=I_{f} R_{1}\left(1-e^{-t / \tau}\right)
$$

where $\tau=R_{1} C_{1}$. The stored charge $Q_{0}$, at time $T_{f}$, is given by the product of capacitance and voltage:

$$
Q_{0}=C_{1} V_{c}=I_{f} \tau\left(1-e^{-T / / \tau}\right) .
$$

This is identical with (24) of Moll et al. It is interesting to note the physical identification of the time constant $R_{1} C_{1}$ of the model with the minority carrier lifetime of the steprecovery diode. The model resistance $R_{1}$ can also be identified with the forward resistance of the diode in the absence of parasitic series resistance.

When the applied bias is reversed, the model becomes as shown in Fig. 1(b), as long as the charge $Q$ is greater than zero. Since $Q$ becomes zero when $V_{c}$ reaches zero, we can compute the duration of charge storage by determining the time when $V_{c}=0$. Again assuming $R_{1} \ll R_{\theta}$, and an initial condition of $V_{c}=Q_{0} / C_{\mathrm{L}}$ when $t=0$, the expression for $V_{c}$ as a function of time is

$$
V_{c}=\left(Q_{0} / C_{1}+I_{r} R_{1}\right) e^{-t / \tau}-I_{r} R_{\mathrm{L}}
$$

where $I_{r}=V_{2} / R_{g}$. The charge-storage time is obtained by solving ( 3 ) for the time $T_{s}$ when $V_{c}=0$. The result is

$$
T_{s}=\tau \ln \left(1+Q_{0} / I_{r} \tau\right) .
$$

Equation (4) is identical with (23) of Moll et al.

If current due to residual charge is neglected [1], at time $T_{8}$ the model becomes simply a capacitance $C_{2}$, and remains so as long as $V_{c}<0$. This capacitance $C_{2}$ can be identified with the depletion-layer capacitance of a reverse-biased diode. The circuit model can be further refined by including the effects of diode contact potential $V_{0}$, and parasitic series resistance $R_{s}$. The resulting circuit model is indicated in Fig. 2.

This piecewise linear model was checked both by programming an analog computer and constructing a model from linear resistors and capacitors and an electronic switch. Typical step-recovery diode waveforms were obtained in each case. One such waveform is shown in Fig. 3. This is the current through a model "diode" in response to a sinusoidal voltage. For this model, $R_{1}$ $=1.5 \mathrm{k} \Omega, C_{1}=0.5 \mu \mathrm{f}, \quad C_{2}=.0005 \mu \mathrm{f}$, and $V_{0}=0$. The input frequency is $200 \mathrm{c} / \mathrm{s}$. Note that $\tau=0.75 \mathrm{~ms}$, which is considerably

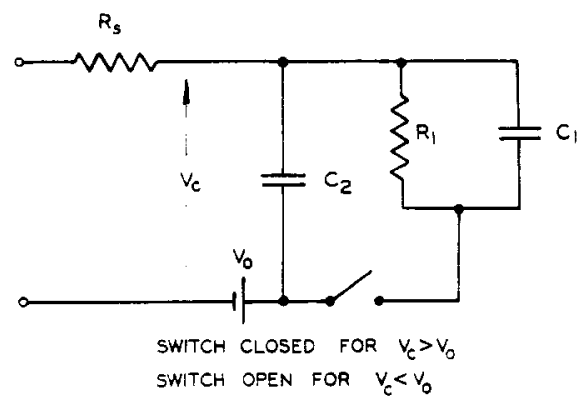

Fig. 2. A piecewise linear model of the step-recovery diode, including contact potential and series resistance.

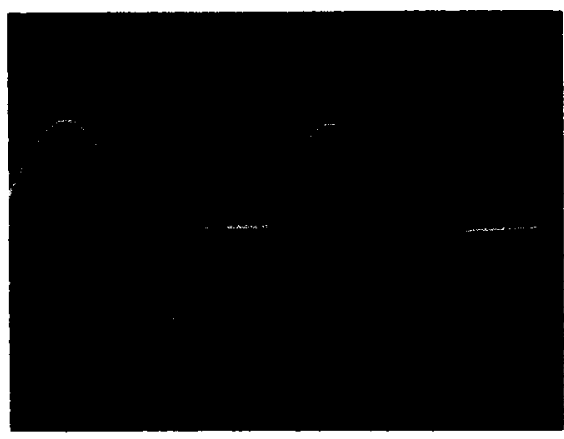

Fig. 3. Typical current waveform obtained with experimental piecewise linear model with sinus oidal applied voltage at $200 \mathrm{c} / \mathrm{s}$.

greater than the lifetime in a typical steprecovery diode, which is on the order of 100 ns. This scaling latitude makes possible the experimental investigation of step-recovery diode action at frequencies much lower than that which must be employed with the actual diode.

On the basis of this model of the steprecovery diode, an interesting comparison can be made between the step-recovery diode and the varactor diode. In the varactor case, the model is a constant series resistance and a voltage-dependent capacitance. The curve of capacitance vs. voltage is smooth, and contains no discontinuities. Under reverse bias, the model of a step-recovery diode is identical to that of the varactor (not withstanding the fact that we have here chosen to treat the depletion layer capacitance of a step-recovery diode as constant in our linearized model). Forward bias operation of a varactor is of ten ignored; at other times some consideration is given to 